VI.

Aus der medicinischen Klinik zu Strassburg i. E.

\title{
Ueber die Kalk- und Magnesiaausscheidung beim Diabetes melitus und ihre Beziehung zar Ausscheidung abnormer Säuren (Acidose) ${ }^{1}$. \\ Von
}

Dietrich Gerhardt und Wilhelm Schlesinger.

Vermehrung des Harnkalkes beim Diabetiker findet sich in der älteren Zeit wiederholt angegeben. Boecker fand 4,23 g Erdphosphate, Benek e Vermehrung des phosphorsauren und oxalsauren Kalkes, $\mathrm{Ne} \mathrm{ubau} \mathrm{er}{ }^{2}$ ) bei einem sechsjährigen Kinde, das an sehwerem Diabetes litt, $0,711 \mathrm{~g}$ phosphorsauren Kalk gegen 0,358 in der Norm. Toralbo ${ }^{3}$ ) will 2,58 pro Tag gefunden haben. v. Noorden ${ }^{4}$ erwähnt bisher unpublicirt gebliebene Versuche van Ackeren's, denen zufolge Kalk- und Phosphorsäure im Harne und in den Fäces eines schweren Diabetikers gegenüber der Einfuhr sich vermehrt erwiesen ${ }^{5}$ ).

Wir gingen davon aus, dass möglicherweise die grosse Kalkausfuhr im Harne mit der Ausseheidung grosser Mengen abnormer Säuren in Beziehung zu bringen sei, und suchten festzustellen, in wieweit die Kalkansscheidung - vorerst im Harne - mit den Ammoniakwerthen parallel laufe, die bekanntlich einen guten Maassstab für den Grad der Acidose abgeben.

1) Eine vorläufige Mittheilung erfolgte auf dem Internat. medicin. Congress zu Moskau im August 1897.

2) Neubauer, Journ. f. prakt. Chemie Bd. LXVII, 1856. S. 76.

3) Toralbo, Centralbl. f. inn. Med. 1890. S. 19.

4) v. Noorden, Pathol. des Stoffwechsels.

5) Auf die seit der Beendigung unserer Arbeit erschienenen Arbeiten von Tennbaum (Zeitschr. f. Biol. Bd. XXXIII) und Moraczewski (Centralbl. f. inn. Med. 1898) sei diesmal bloss hingewiesen. 
Dass grosse Säuremengen, dem Organismus zugeführt, den Kalkwerth des Harnes steigen machen, ist für die Zufuhr anorganischer Säuren per os durch Schetelig und Gaethgens festgestellt. Ersterer ${ }^{1)}$ beobachtete nach Zufahr von Acid. muriat. unter sonst gleichen Bedingungen ein Ansteigen des $\mathrm{CaO}$ von 400 auf $500 \mathrm{mg}$ pro die. Bei des Letzteren ${ }^{2}$ ) Versuchen am Hunde nahmen nach Einbringen von $7 \mathrm{~g}$ Schwefelsäureanhydrid Kalk - und Ammoniakwerthe des Harns gleichsinnig zu. Desgleichen stieg in Rüdel's ${ }^{3}$ ) Versuchen bei einem $3^{1 / 2}$ jäbrigen Mädchen nach Darreichung von $3 \mathrm{ccm}$ Acid. muriat. dil. der Kalk von 0,049 auf 0,059 an, beim Hunde unter 4,0 Acid. muriat. concentr. von 0,033 auf 0,066 pro die. Weniger bestimmt lauten die Angaben über vermehrte Kalkausfuhr bei Zuführung organischer Säure. Solche Versuche wurden mit Milchsäure wiederholt mit Rücksicht auf die alte Anschauung angestellt, dass bei Rachitis gesteigerte Milchsäurebildung Ursache einer Lösung von Knochenkalk werde. Teissier ${ }^{4}$ ) will thatsächlich in solchen Versuchen „Kalkvermehrúng der Abgänge" beobachtet haben. Dagegen ergaben die sehr sorgfältigen Versuche von Heiss 5 ) bei monatelang fortgesetzter Verabreichung von 4-9 g reiner Milehsäure weder gesteigerte Ausfuhr von Kalk in Harn und Fäces, noch ein Aermerwerden der Knochen an dieser Base ${ }^{6}$ ). Da indessen die Milehsäure, wie man seit Wöhler weiss, vom Magen aus aufgenommen, den Körper nicht mehr als solche, sondern zu Kohlensäure verbrannt, verlässt, ist von ihrer Einfuhr ein Ansteigen der Kalkwerthe nicht zu erwarten. Bemerkenswerther erscheint uns Sohetelig's Angabe, dass der Morgenharn die grössten Kalkmengen enthalte mit Rücksicht darauf, dass er erfahrungsgemäss den höchsten Säuregrad besitzt.

Zufuhr von Alkalien drückt den Kalkwerth des Harnes herab. So enthält der alkalische Harn der Pflanzenfresser bloss Spuren vọn

1) Schetelig, Virch: Archiv Bd. LXXXII. S. 43\%. 1880.

2) Ga ethgens, Zeitschr. f. physiol. Chemie Bd. IV. S. 36. 1880.

3) Rüdel, Archiv f. experiment. Pathol. u. Pharmakol. Bd. XXXIII. S. 79.

4) Teissier, cit. bei Heiss, s. Anm. 5 .

5) Heiss, Zeitschr. f. Biol. Bd. XII. S. 151.

6) Zülzer (,,Untersuchungen über die Semiologie des Harnes“, Berlin 1884, S. 101) fand bei einem mit Stärke gefütterten Hunde viel Harnkalk und glaubt dies auf Milchsäurebildung im Darme beziehen zu sollen.

Senator (,Ueber die Kalkausscheidung im Harne bei Lungenschwindsucht", Charité-Annalen 1882, Bd. VII. S. 397) spricht die Hypothese aus, dass bei der Umwandlung von gelbem in rothes Mark reichlicher producirte Milchsäure Knochenkalk löse. 
Ueber die Kalk- und Magnesiaausscheidung beim Diabetes melitus u. s. w. 85

Kalk wie von Phosphorsäure. Beckmann ${ }^{1}$ sah nach $5 \mathrm{~g}$ Natr. earbon. den Kalkwerth des Harnes beim Mensehen absinken. In Bertram's ${ }^{2}$ ) Versuchen fiel er nach Verabreichung von $40 \mathrm{~g}$ eitronsaurem Kali von 0,5 auf 0,285 . Ein solcher Einfluss des citronsauren Salzes wurde indessen von Beckman n vermisst.

Wir benutzten das bekannte Verhalten des Natriumbicarbonates zur Ammoniakausscheidung, um diese steigen oder fallen zu machen, und begnügten uns vorerst festzustellen, in welchem Maasse das Verhalten des Harnkalkes mit dem Ansteigen und Abfallen des Ammoniaks parallel läuft.

Der 26 jährige, durch die Arbeiten von Weintraud bekannte Schneider T. ${ }^{3}$ ) leidet an Diabetes sehwerer Form. Einmal wird er erst bei starker Einschränkung der Eiweisszufuhr zuckerfrei, dann scheidet er seit 3 Jahren unausgesetzt enorme Mengen von Aceton, Acetessigsäure und $\beta$-0xybuttersäure aus ${ }^{4}$ ). Seit Monaten besteht seine tägliche Diät aus $200 \mathrm{~g}$ Fleisch (gekocht gewogen), 2 Eiern, $150 \mathrm{~g}$ Speck, $75 \mathrm{~g}$ Butter, $200 \mathrm{~g}$ Gemüse (Spinat), 1/2 Liter Wein. Dazn erhält er $20 \mathrm{~g}$ Natr. bic. pro die. Bei dieser Diät bleibt sein Körpergewicht annähernd constant, die tägliche Zuckerausscheidung beträgt gegen $30 \mathrm{~g}$. Da es uns für unseren Versuch wichtig ersehien, vorerst die seit Monateu wirksamen Ernährungsbedingungen festzuhalten, verzichteten wir darauf, den Harn zuckerfrei zu machen. Unsere Versuche wurden jedesmal in der Weise angeordnet, dass in einer Vorperiode $\mathrm{NH}_{3}$ - und Ca-Werth des Harnes festgestellt, in einer 2. Periode (8 Tage) das Alkali weggelassen wurde. Das Deficit an Alkali kam im Ansteigen der $\mathrm{NH}_{3}-$ Werthe prompt zum Ausdrucke. In einer 3. Periode wurden wiederum $20 \mathrm{~g}$ Natr. bic. gegeben. Gleichzeitig angestellte Bestimmungen der Erden sollten die Frage beantworten, ob auch sie gleichsinnig mit dem $\mathrm{NH}_{3}$ im Harne ansteigen.

Ammoniak wurde nach der Methode von Schlösing bestimmt. Ausserdem wurde der Gesammtstickstoff nach Kjeldahl, die Phosphorsäure durch Titriren mit Uranacetat, die auf saures Phosphat entfallende Menge derselben nach Freund (Ausfällen der neutralen Phosphate mit Chlorbarium in Substanz und Titriren der Phosphor-

1) Beckmann, bei Stadelmann, "Linfluss der Alkalien auf den menschlichen Stoffwechsel", Stuttgart 1890. S. 52.

2) Bertram, Zeitschr. f. Biol. Bd. XIV. S. 335. 1878.

3) Naunyn, Der Diabetes mellitus. Wien 1898. Fall 53, bez. Fall 91.

4) Der Kranke starb ein Jahr nach dem Abschlusse unserer Untersuchungen im Coma. 
säure im Filtrate), der Zucker zumeist durch Titriren nach Fehling und Polarisiren im Halbschattenapparate, Aceton nach Messinger bestimmt ${ }^{1}$ ). Aus der nach dem Vergähren des Harnes mit Hefe am Saccharimeter abgelesenen Linksdrehung konnte die Menge der an diesem Tage ausgeschiedenen Oxybuttersäure gesehätzt werden.

In einer Versuchsreihe, die nur zu unserer vorläufigen Orientirung diente, trachteten wir über die Menge der ausgeschiedenen Erden überhaupt durch Bestimmung der an Phosphorsäure gebundenen Erden ein Urtheil zu gewinnen (Boecker, Beneke).

In einer Harnportion (20 ccm) wird die Gesammtphosphorsäure durch Titriren mit essigsaurem Uran gegen Cochenilletinctur bestimmt. Eine andere, grössere Portion wird mit einigen Tropfen Ammoniak bis zum Auftreten eines deutlichen Niederschlages versetzt, gelinde erwärmt, wobei sich der gelatinöse Niederschlag verdichtet und zu Boden setzt, darnach filtrirt. Vom Filtrate werden wieder $20 \mathrm{ecm}$ abgemessen und darin die $\mathrm{P}_{2} \mathrm{O}_{5}$ titrimetrisch bestimmt. Die Differenz zwischen beiden Werthen ergiebt die Menge der ursprünglich an alkalische Erden gebundenen und als basisches Calciumphosphat und phosphorsaure Ammoniakmagnesia gefällten Phosphorsäure und damit ein Maass für erstere selbst. Auch in den folgenden Versuchen wurde neben Kalk und Magnesia auch die an alkalische Erden gebundene Phosphorsäure bestimmt; dabei ergab sich, dass der so erhaltene $\mathrm{P}_{2} \mathrm{O}_{5}$-Werth thatsächlich ein Bild der $\mathrm{Ca}+\mathrm{Mg}$ Werthe annähernd widerspiegelt. Vermuthlich würde durch Einengung der Fehlerquellen einmal durch Titriren einer grösseren Harnmenge, anderseits durch Anwendung einer-verdünnteren Uranlösung (wir benutzten solche vom Titer $1 \mathrm{cem}=5 \mathrm{mg} \mathrm{P}_{2} \mathrm{O}_{5}$ ) hierin noch weitergehender Parallelismus festzustellen sein.

Von der ausführlichen Wiedergabe dieses Versuches absehend, heben wir aus der Tabelle hervor, dass die $\mathrm{NH}_{3}$-Werthe nach dem Aussetzen des Alkali von $2 \mathrm{~g}$ pro die auf $5 \mathrm{~g}$.anstiegen, um bei erneuerter Darreichung des Salzes zum ursprünglichen Werthe abzufallen. Dementsprechend stieg auch der Werth für die an Ca und $\mathrm{Mg}$ gebundene $\mathrm{P}_{2} \mathrm{O}_{\overline{5}}$ von durchschnittlich 0,8 auf $1,2 \mathrm{~g}$ an.

\section{Versuch. (S. nebenstehende Tabelle I.)}

Tabelle I giebt den einen Monat später in gleicher Weise ausgeführten 2. Versuch wieder. Der Kranke erhielt durch 4 Tage $20 \mathrm{~g}$ Natr. bic.; in der II. Periode, die 8 Tage umfasst, wurde das Alkali weggelassen; darauf folgt die III. (viertägige) Periode mit neuerlicher Verabreichung. In jeder Periode wurden an einzelnen Tagen Kalkbestimmungen ausgeführt. (Fällen mit oxalsaurem Ammon, Glühen im Gebläse bis zur Gewichtsconstanz, Wägen als $\mathrm{CaO}$ ).

1) Neubauer und Vogel, Harnanalyse. 
Ueber die Kalk- und Magnesiaausscheidung beim Diabetes melitus u. s. w. 87

\begin{tabular}{|c|c|c|c|c|c|c|}
\hline 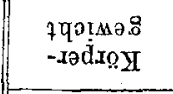 & 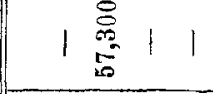 & 11 & | & 1 & 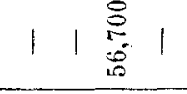 & 111 \\
\hline 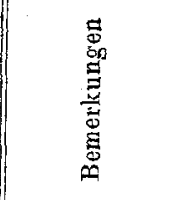 & 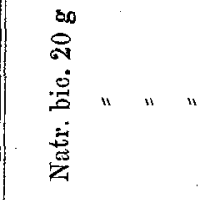 & 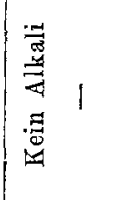 & 1 & 1 & 1111 & 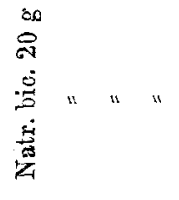 \\
\hline$\nexists$ & 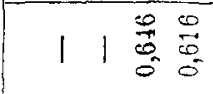 & 1 害 & 1 & 量 & 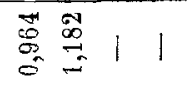 & $\left|\begin{array}{l}0 \\
\mathbb{G} \\
0\end{array}\right|$ \\
\hline 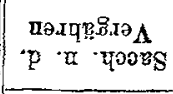 & 1118 & 11 & 1 & $\stackrel{\infty}{\infty}$ & $\begin{array}{llll}\infty & 0 & \infty & 0 \\
0 & 0 & 0 \\
1 & 1 & 1 & 0 \\
0\end{array}$ & {$\left[\begin{array}{llll}0 & 0 & 0 & \\
0 & 0 & 0 & 0 \\
1 & 1 & 1 & 0 \\
1 & 1 & 1\end{array}\right.$} \\
\hline$z||_{20}^{\infty}$ & 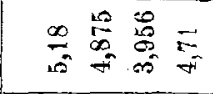 & 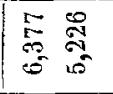 & $\begin{array}{l}\overrightarrow{1} \\
\substack{++}\end{array}$ & $\underset{F}{\stackrel{2}{*}}$ & 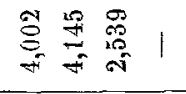 & 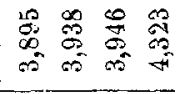 \\
\hline $\mathfrak{G}^{\infty}$ & 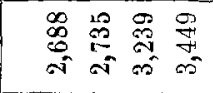 & 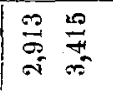 & $\stackrel{109}{=}$ & $\ddot{E}_{0}^{\circ}$ & 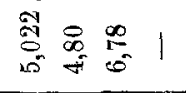 & 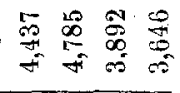 \\
\hline$\left.z\right|_{0} ^{\infty}$ & 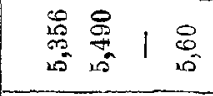 & 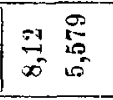 & $\begin{array}{l}\overrightarrow{\mathbb{N}} \\
\text { is } \\
\text { is }\end{array}$ & \begin{tabular}{c}
$\infty$ \\
\multirow{2}{*}{} \\
$\infty$
\end{tabular} & 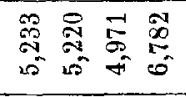 & 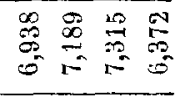 \\
\hline 氙 & 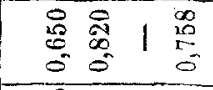 & $\mid \begin{array}{ll}0 & 0 \\
0 & 0 \\
0 & 0 \\
0 & 0 \\
0\end{array}$ & 足 & $\%$ & 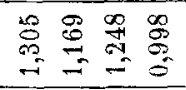 & 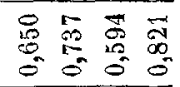 \\
\hline 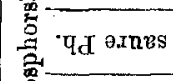 & o 11 क & $\stackrel{n}{8} \bar{\pi}$ & $\stackrel{2 \pi}{9}$ & $\begin{array}{l}12 \\
00 \\
68\end{array}$ & 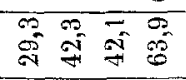 & $\begin{array}{l}0 \\
0 \\
0\end{array}$ \\
\hline 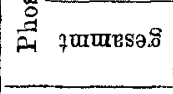 & 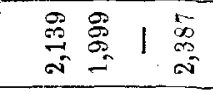 & 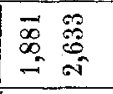 & $\frac{60}{60}$ & 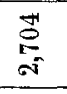 & 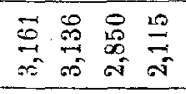 & 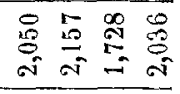 \\
\hline$z$ & 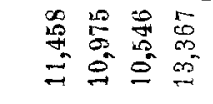 & 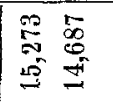 & $\begin{array}{l}\infty \\
\infty \\
0 \\
0\end{array}$ & $\stackrel{g}{g}$ & 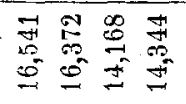 & 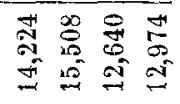 \\
\hline & $111 \frac{5}{6}$ & $\stackrel{\infty}{\Rightarrow}$ & 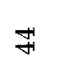 & 5 & 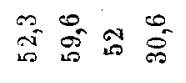 & 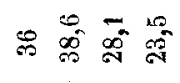 \\
\hline 荵 & $111=$ & 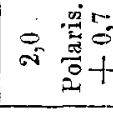 & 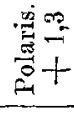 & $\vec{z}$ & $\dddot{m}$ & $\stackrel{\infty}{=}=$ \\
\hline 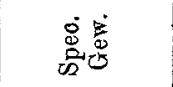 & 令 & 竎 突 & 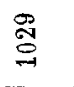 & 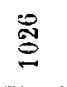 & 홍 & 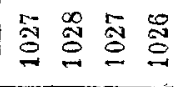 \\
\hline 离 & 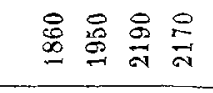 & 悉总 & 胥 & 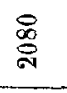 & 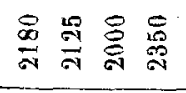 & 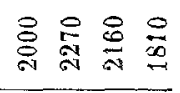 \\
\hline 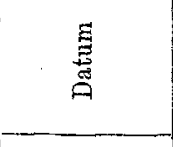 & 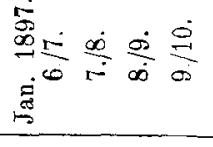 & 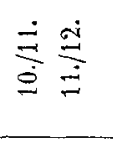 & 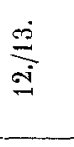 & $\underset{\stackrel{i}{*}}{\stackrel{i}{i}}$ & 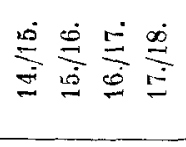 & 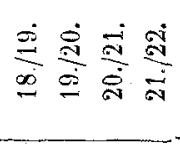 \\
\hline & $\partial$ & & & & & \\
\hline
\end{tabular}


Während der I. Periode (6.-10. Jan.) befand sich der Kranke annähernd im Stickstoffgleichgewichte. Die Berechnung des N-geehaltes der Nahrung ergiebt etwa $14 \mathrm{~g}{ }^{1}$ ). Ebenso hoch dürfte die durchsehnittliche Ausseheidung mit dem Harne und mit den Fäces gewesen sein. Während der alkalifreien Periode steigen die N-Werthe im Harne allein auf $16,5 \mathrm{~g}$ pro die an, um sich in der letzten Periode bei neuerlicher Darreichung von Natr. bic. wieder langsam den früheren Werthen zu nähern.

Gleiche Verhältnisse während des ersten und des später zu beschreibenden 3. Versuches bewiesen uns, dass wir nicht darauf rechnen durften, diesen Kranken dauernd im Stickstoffgleichgewichte zu erhalten. Zudem zeigten sich schon bei der durch viele Monate eingehaltenen Standarddiät ( $+20 \mathrm{~g}$ Natr. bic.), wobei der Kranke sein Körpergewicht annähernd bewahrte, die Stickstoffzahlen sehwankend. Auf diesen Umstand wollen wir später noch zurtickkommen.

Die Ammoniakzahlen bewegen sich während der I. Periode um Werthe von $3 \mathrm{~g}$, während der II. Periode wird die hohe Ausscheidung von $6,8 \mathrm{~g}$ pro die erreicht, in der III. Periode sinken sie wieder ab.

Die täglich mit dem Harne ausgeschiedene Kalkmenge beträgt am 3. und 4. Tage der I. Periode 0,646 und $0,616 \mathrm{~g}$, nach der Entziehung des Alkali steigt sie auf fast das Doppelte an (am 15./16. 1,182), eine Bestimmung während der III. Periode ergiebt wieder $0,649 \mathrm{~g}$.

Demnach erschien ein Parallellaufen der Werthe für die beiden Basen zumindest sehr wahrscheinlich.

Um dem Einwande zu begegnen, es hätte an einzelnen Tagen Kalkretention stattgefunden, und um über die Gesammtausscheidung: des Kalkes ein Urtheil zu gewinnen, wurde wieder einen Monat später ein dritter gleich angeordneter Versuch an dem gleichen Patienten mit fortlaufenden Kalk- und Magnesiabestimmungen im Harne und in den Fäces angestellt. (S. Tabelle II.)

Gleichzeitig brachten wir einen Nichtdiabetischen in gleiche Ernährungsverhältnisse, um auch an ihm die Ausscheidung der beiden Basen zu studiren. (S. Tabelle III S. 90.)

1) $200 \mathrm{~g}$ Fleisch (gekocht)

2 Eier

$200 \mathrm{~g}$ Spinat

$150 \mathrm{~g}$ Speck

$75 \mathrm{~g}$ Butter

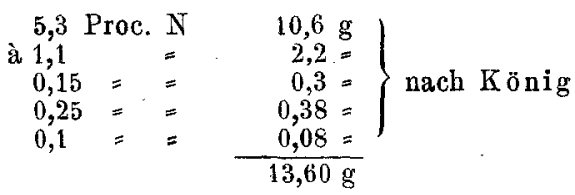


Ueber die Kalk- und Magnesiaausscheidung beim Diabetes melitus u. s. w. 89

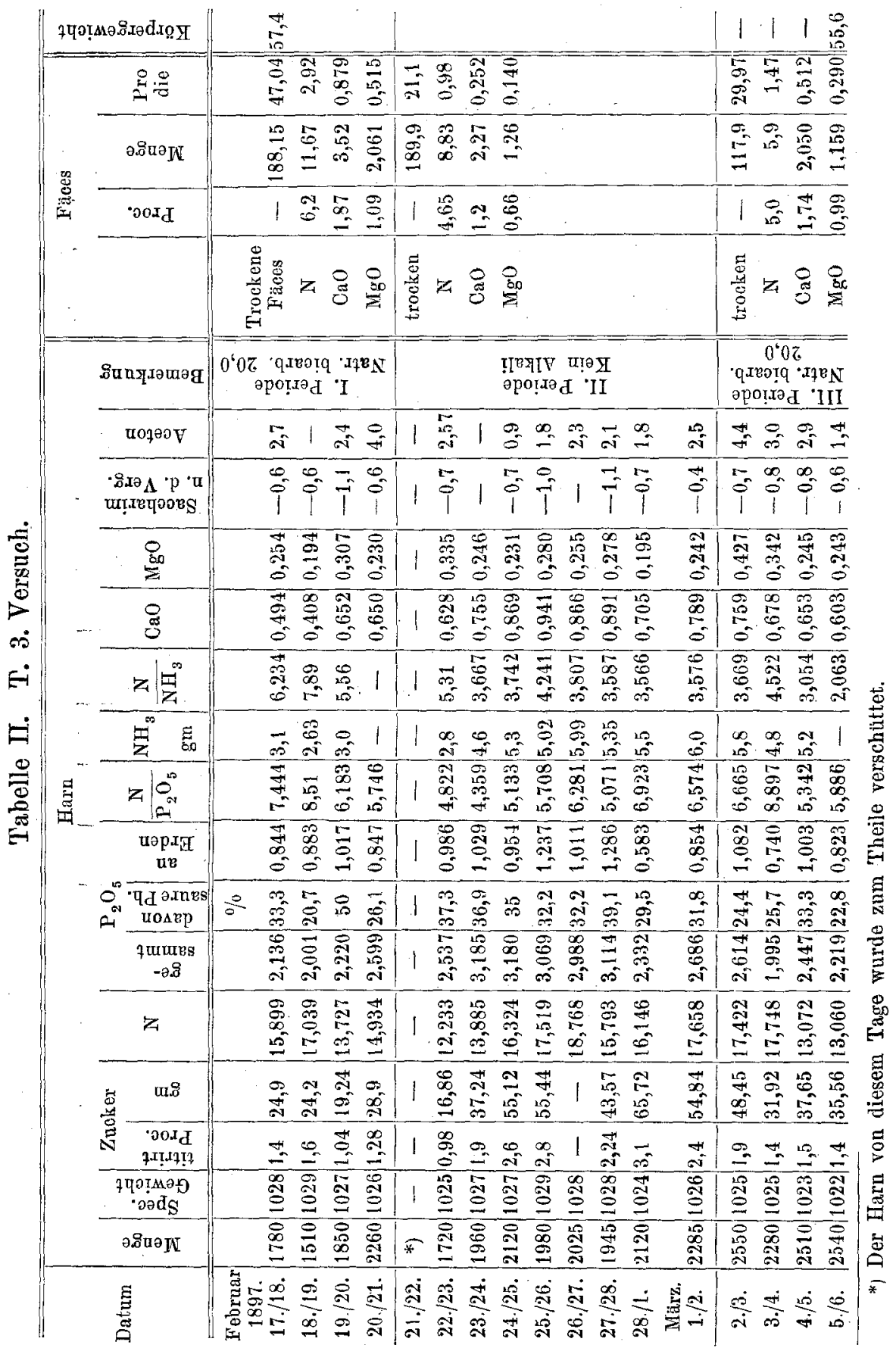




\begin{tabular}{|c|c|c|c|}
\hline III. Periode & II. Periode & I. Periode & \\
\hline 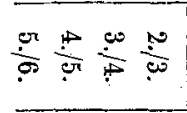 & 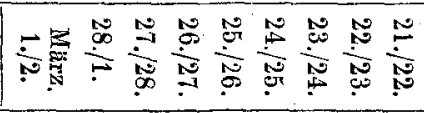 & 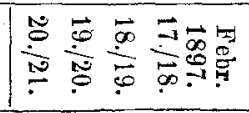 & 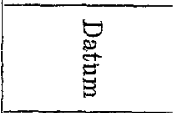 \\
\hline 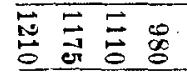 & 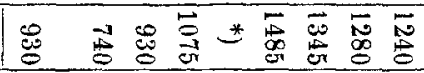 & $\begin{array}{l}\overrightarrow{0} \overrightarrow{0} \\
\dot{0}\end{array}$ & Menge \\
\hline 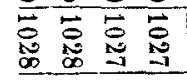 & 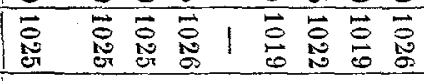 & 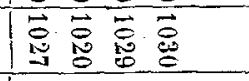 & $\begin{array}{c}\text { Spec. } \\
\text { Gewicht }\end{array}$ \\
\hline 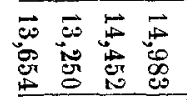 & 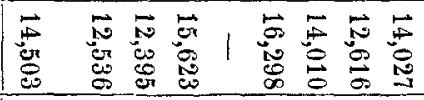 & 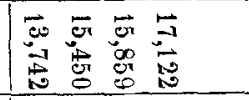 & $z$ \\
\hline 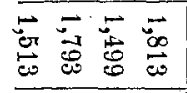 & 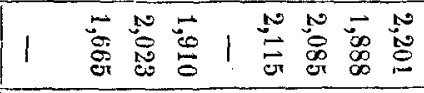 & 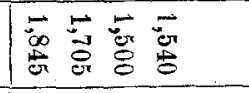 & $\begin{array}{l}0 \\
0 \\
0.02 \\
0\end{array}$ \\
\hline 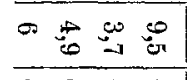 & 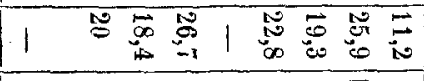 & $\overline{0} \vec{i} \vec{\infty} \overrightarrow{i v} \div$ & $\begin{array}{l}\text { davon } \\
\text { s. Ph. }\end{array}$ \\
\hline 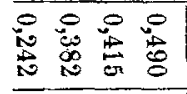 & 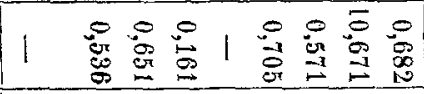 & 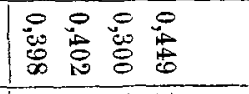 & $\begin{array}{c}\text { an } \\
\text { Erden }\end{array}$ \\
\hline 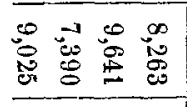 & 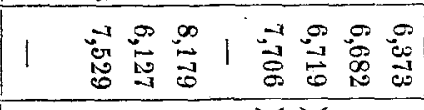 & 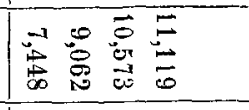 & $\begin{array}{lll}10 \\
0 \\
0 \\
0\end{array}$ \\
\hline 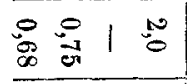 & 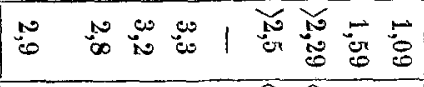 & $5: 080$ & 亘 \\
\hline 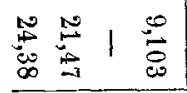 & 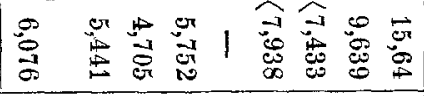 & 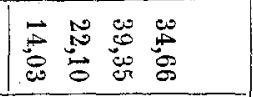 & 离| \\
\hline 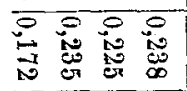 & 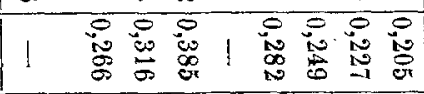 & 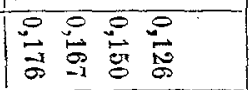 & $\stackrel{8}{0}$ \\
\hline 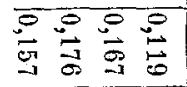 & 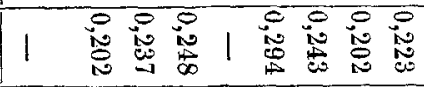 & 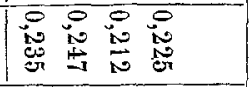 & 高 \\
\hline $\begin{array}{llll}1 & 1 & 1 & 1 \\
0 & 0 & 0 & 0 \\
= & = & 0 & 0 \\
\end{array}$ & 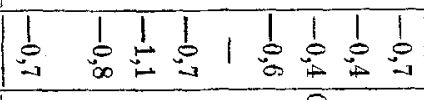 & $\begin{array}{llll}1 & 1 & 1 \\
0 & 1 & 0 & 0 \\
0 & 0 & 0 & 0 \\
\end{array}$ & Saccharim. \\
\hline 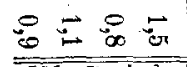 & 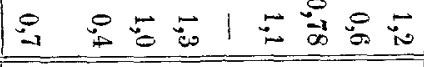 & 1111 & Aceton \\
\hline \multirow[t]{5}{*}{$\begin{array}{c}\text { II. Periode } \\
\text { Natr. bicarb. } \\
20,0 \\
\end{array}$} & $\begin{array}{l}\text { II. Periode } \\
\text { Kein Alkali }\end{array}$ & $\begin{array}{c}\text { I. Periode } \\
\text { Natr. bicarb. } 20, \mathbf{0}\end{array}$ & Bemerkungen \\
\hline & & 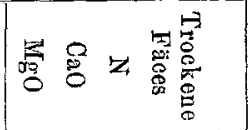 & \\
\hline & & $\mid \begin{array}{lll}0 & 0 & 0 \\
\infty & 0 & 0 \\
0 & 0 & 0\end{array}$ & Proe. \\
\hline & & $\begin{array}{llll}5 & 0 \\
0 & 0 & 0 & 0 \\
0 & 0 & 0 \\
0 & 0 & 0 & 0 \\
0 & 0 & 0\end{array}$ & 疍 \\
\hline & & $0 \begin{array}{lll}0 & 0 \\
0 & 0 & 0 \\
0 & 0 & 0 \\
0\end{array}$ & $8:-4$ \\
\hline 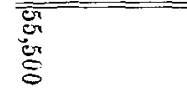 & 跑 & 吾 & $\begin{array}{l}\text { Körper- } \\
\text { gewioht }\end{array}$ \\
\hline
\end{tabular}


Ueber die Kalk- und Magnesiaausscheidung beim Diabetes melitus u. s. w. 91

W., 27 Jahre alt, leidet an Syringomyelie mässig vorgeschrittenen

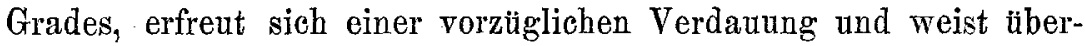
haupt keinerlei Störungen im Bereiche der vegetativen Funktionen auf. Die an ihm gewonnenen Zahlen sind in Tabelle III niedergelegt.

Unter dem Einflusse der reinen Fleischfettdiät wurden in seinem Harne schon am 3. Tage ('dem ersten unseres Versuches) Aceton und Acetessigsäure in grosser Menge nachweisbar. Das Polarimeter zeigte eine Ablenkung nach links um -0,3 Grade, die im weiteren Verlaufe bis auf $\mathbf{- 1 , 1}$ anstieg. Als Ursache dieser bedeutenden Linksdrehung wurde $\beta$-Oxybuttersäure ermittelt. Wir werden am Schlusse unserer Arbeit auf diesen merkwirdigen Fund bei einem Nichtdiabetischen zurückkommen.

Vergleichen wir zunächst die Kalkzahlen, so fällt ebenso wie in Tabelle I die absolute Höhe der Werthe beim Diabetiker T. gegenüber den Zahlen bei W. auf. Erstere sind in Periode I und III drei- bis viermal so hoch wie letztere. Gleicherweise sind aber anch die $\mathrm{NH}_{3}$-Werthe bei T. schon während der Alkalidarreichung ausserordentlich hoch ( $3 \mathrm{~g}$ in beiden Versuchen) zu einer Zeit, wo sich der Kranke, sowohl den täglich ausgeschiedenen Stickstoffzahlen nach zu urtheilen, als nach dem durch Jahre annähernd gleich gebliebenen Körpergewicht, in einer relativen Gleichgewichtslage befunden baben muss.

Als aber das Alkali entzogen wurde (II. Periode), stiegen die Ca-Werthe auf fast $0,95 \mathrm{~g}$ pro die an (am 26. Februar), im Mittel sich constant um $0,8 \mathrm{~g}$ erhaltend, ebenso wie sich die Ammoniakzahlen nach raschem Ansteigen um Werthe von $5^{1 / 2} \mathrm{~g}$ bewegen.

Auffallend ist in diesem Versuche (3) nur das prompte Abfallen der Ca-Werthe in der Nachperiode, während die $\mathrm{NH}_{3}$-Zahlen diesmal nicht gleich rasch ihrer ursprünglichen Grösse zustreben.

Bei der Controlperson beobachteten wir das Gleiche, nur in weniger hohem Grade.

Als Ausdruck der durch reine Fleischfettdiät auch hier zustande gekommenen Acidose finden wir auch bei ihm abnorm hohe Ammoniakwerthe (um $3 \mathrm{~g}$ während der Periode II). Durch Verabreichung ron Natr. bic. (I. und III. Periode) gelingt es hier allerdings, die $\mathrm{NH}_{3}$ Ausscheidung mit dem Harne zur Norm herabzudrücken. Vollkommen parallel mit dem Steigen und Sinken des Ammoniaks erheben sich auch die ursprünglich niedrigen $\mathrm{Ca}$-Werthe von 0,17 allmählich bis 0,4 am 26./27., um bei neuerlicher Darreichung des Alkali wieder zu ibrer anfänglichen Grösse abzusinken. 
Am 28. Jan. und am 1. Februar sinken Ammoniak und Kalk um ein Geringes $a b$, doch wurde am letzteren Tage auch auffallend wenig Aceton ausgeschieden.

Hier scheint uns der Ort, einem Einwande zu begegnen, der gegen unsere Versuchsanordnung erhoben werden könnte.

Man könnte sagen, dass das Ansteigen und Absinken der Kalkwerthe im Harne nicht der Ausdruck complieirter, durch die Säuerung bedingter Alkalescenzverhältnisse in den Geweben ist, sondern dass durch Zuführung von Natriumbicarbonat ein Theil des Kalkes im Darmrohre noch vor seiner Resorption direct gefällt und damit dieser entzogen wurde. Bekanntlich ist das neutrale Calciumphosphat fast unlöslich, das saure Salz viel leichter löslich. Tereg und A n nold ${ }^{1}$ ), die darüber genaue Versuche anstellten, fanden bei Fleischfettfütterung beim Hunde das Verhältniss des im Harne ausgeschiedenen Kalkes zu dem in den Fäces wie 1:5. Wurde nun drei- oder zweibasisches Calciumphosphat dem Futter zugefügt, so wandelte sich dieses Verhältniss in 1:502) und 1:86. Dagegen wurde es 1:25 bei Verabreichung des sauren (einbasischen) Salzes als Ausdruck besserer Resorption des letzten. Nur als solches (oder als complicirtere, gleichfalls saure Kalkeiweissverbindung: ${ }^{3}$ ) gelangt wohl auch unter normalen Verbältnissen Kalk von der Darmwand aus zur Resorption. Aus dem eingeführten Natr. bicarb. könnte nun, sofern es den Magen unverändert passirt, im Darme dureh Dissociation der Kohlensäure fixes Alkali entstehen, das die zur Resorption bestimmten saueren Kalkverbindungen noch vorher als neutrales Calciumphosphat fällen würde. Man könnte unter solcher Annahme die niedrigeren Kalkwerthe im Harne während der Perioden der Alkalizufuhr auf solche im Darme vor sich gehende Ausfällung des Kalkes beziehen, die mit dem Säuerungsvorgange in den Geweben nichts zu thun hat. Diese Auffassung könnte durch den schon erwähnten vereinzelten Befund unterstïtzt werden, dass bei unserem 3. Versuche an $\mathrm{T}$. (Tabelle III) die Ca-Werthe bei wiederholter Verabreichung des Alkali absinken, während die $\mathrm{NH}_{3}$-Werthe hoch bleiben.

Abgesehen von der Unwahrscheinlichkeit, dass bei sonst guter Verdauung nennenswerthe Alkalimengen der Neutralisation durch.

1) Tereg und Arnold, Pflüger's Archiv Bd. XXXII. S. 122.1883.

2) Die anscheinend bessere Resorption des dreibasischen Salzes berechnet sich aus dem Durchschnitte einer Reihe von Tagesbestimmungen, von denen eine einzelne einen ganz besonders hohen Werth aufweist. Die übrigen Zahlen entsprechen den für das zweibasische Salz gefundenen.

3) C. Voit, Physiol. des Stoffwechsels, S. 371. 
Ueber die Kalk- und Magnesiaausscheidung beim Diabetes melitus u. s. w. 93

die Salzsäure des Magens entgehen, steht dieser Einwand in entschiedenem Widerspruche mit dem deutlich zu beobachtenden langsamen Ansteigen des Harnkalkes, vor allem auf Tabelle III (W.). Die Werthe werden ganz allmählich höher, um erst am 27. Februar, also 6 Tage nach dem Aussetzen des Alkali, ihren Höhepunkt zu erreichen, zu einer Zeit, wo das eingegebene Natriumbicarbonat, ent. sprechend seiner grossen Löslichkeit, den Organismus längst verlassen haben musste.

Daza kommt, dass der bei $W$. erreichte höehste Werth höher liegt, als nach den aus der Litteratur vorliegenden Angaben ${ }^{1)}$ bei einer recht kalkarmen Nahrung zu erwarten wäre.

Aehnlich langsames Ansteigen zeigt die an Erden gebundene $\mathrm{P}_{2} \mathrm{O}_{5}$ auf Tabelle I (höchster Werth 6 Tage nach dem Aussetzen des Alkali). Desgleichen erreichen die Kalkzahlen der Tabelle II erst am 5. Tage ihren Höhepunkt.

Ein Parallellaufen der Stickstoff- und Kalkzahlen des Harnes ist nicht nachweisbar. In folgender Tabelle (IV) sind die Durchschnittswerthe für die einzelnen Versuche und Perioden nebeneinander gestellt.

Tabelle IV.

\begin{tabular}{|c|c|c|c|c|c|c|}
\hline \multirow{2}{*}{ Periode } & \multicolumn{2}{|c|}{ T. 2. Versuch } & \multicolumn{2}{|c|}{ T. 3: Versuch } & \multicolumn{2}{|c|}{ W } \\
\hline & $\mathrm{N}$ & $\mathrm{Ca}$ & $\mathrm{N}$ & $\mathrm{Ca}$ & $\mathrm{N}$ & $\mathrm{Ca}$ \\
\hline I. & 11,587 & $\begin{array}{c}\text { niedrige } \\
\text { Werthe }\end{array}$ & 15,399 & 0,551 & 15,543 & 0,155 \\
\hline II. & 15,234 & $\begin{array}{c}\text { hobe } \\
\text { Werthe }\end{array}$ & 16,041 & 0,805 & 14,001 & 0,276 \\
\hline III. & 13,837 & $\begin{array}{c}\text { niedrige } \\
\text { Werthe }\end{array}$ & 15,325 & 0,673 & 14,085 & 0,217 \\
\hline $\begin{array}{l}\text { Ganzer } \\
\text { Versuch }\end{array}$ & 13,973 & 一 & 15,702 & 0,709 & 14,151 & 0,231 \\
\hline
\end{tabular}

Vergleichen wir zunächst die Durchschnittszahlen für die Controlperson W., so ergiebt sich, dass in der I. Periode einem hohen NWerthe ein viel niedrigerer Ca-Werth entspricht als in der folgenden Periode. Ebenso steht in der II. Periode eine etwas höhere $\mathrm{N}$-Zahl einer niedrigeren Ca.Zahl gegenüber. Im 3. Versuche T. kann eine Mehrausgabe von weniger als $0,7 \mathrm{~g} \mathrm{~N}$ in der II. Periode gegenüber den beiden Alkaliperioden unmöglioh zu der entsprechenden um die Hälfte höheren Ca-Zahl in ursächliche Beziehung gebracht werden. In der I. Periode, also unter gleichen Bedingungen, scheidet T. bei

1) Neubauer und Vogel, Analyse des Harnes. 
gleich viel Stickstoff fast viermal so viel Kalk aus, wie W.; der um ein geringes höheren durchschnittlichen $\mathrm{N}$-Ausscheidung (für den ganzen Versuch) T.s steht eine Kalkziffer gegenüber, welche die der Controlperson um das Dreifache übertrifft.

Auch die einzelnen 'Tageswerthe lassen jede Uebereinstimmung' vermissen. Man vergleiche beispielsweise

$\begin{array}{rlcc} & & \mathrm{N} & \mathrm{Ca} \\ \text { auf Tabelle III } & \text { 25. Febr. } & 16,3 & 0,28 \\ & 28 . \quad= & 12,4 & 0,32 \\ \text { oder auf Tabelle II } & 26 . \quad \Rightarrow & 17,5 & 0,94 \\ & \text { 2. März } & 17,7 & 0,79\end{array}$

Bei den N-Werthen unserer Tabellen verweilend, sehen wir, dass bei W. die Entziehung des Alkali ohne wesentlichen Einfluss auf die N-Ausfuhr mit dem Harne blieb. In der I. Periode (Tabelle III) sinkt die hohe - wohl noch als Einstellungswerth zu bezeichnende - N-Zahl von $17,1 \mathrm{~g}$ am ersten auf $13,5 \mathrm{~g}$ am letzten Tage; den gleichen Werth finden wir an den beiden letzten Tagen der II. Periode wieder. Er entspricht wohl der Gleichgewichtslage. (Der Kranke hatte am Ende der 2. Woche wieder so viel an Körpergewicht zugenommen, als er in der 1. Woche verloren hatte.) Während der Entziehung des Alkali steigt der. Werth allerdings an 2 Tagen bis $16 \mathrm{~g}$. Die Durchsehnittszahl bleibt indessen in der II. und III. Periode die gleiche (Tabelle IV); die höhere Durchschnittszahl der I. Periode $(\mathbf{1 5}, 5)$ erklärt sich aus den hohen $\mathrm{N}$-Werthen der ersten 3 Tage, wo die Gleichgewichtslage noch nicht erreicht war.

Anders bei T. Einmal finden wir die aus dem ganzen Versuche (3) berechnete Durchschnittszahl höher als bei W. bei gleicher Diät. Letzterer scheidet $14,151 \mathrm{~g} \mathrm{~N}$ pro die mit dem Harne aus, T. 15,702 g. Dagegen beträgt die durchschnittliche Stickstoffausfuhr mit dem Harne auch bei T. im 2. Versuche bloss gegen $14 \mathrm{~g}$. Andrerseits hat die Entziehung des Natr. bic. bei dem schweren Diabetiker T. eine deutliche Steigerung der N-Zahl des Harnes zur Folge. Am klarsten erhellt dies aus dem 2. Versuche (Tabelle I), zu dessen Beginne und Ende (I. und III. Periode) er sich rücksichtlich der Stickstoffausscheidung offenbar in einem relativen Gleichgewichtszustande befand. Der N-Werth beträgt während der I. Periode im Durchschnitte 11,6 g, steigt nach der Entziehung des Alkali auf 15,2, um nach neuerlicher Verabreichung wieder auf 13,8 abzusinken. Jede einzelne N-Zahl der mittleren Periode ist höher, als die Zahlen der Vor- und Nachperiode. 
Ueber die Kalk- und Magnesiaausscheidung beim Diabetes melitus u. s. w. 95

$\mathrm{Zu}$ Beginn des 3. Versuches war bei T. - vermuthlich als Folge der vorausgegangenen wiederholten Entziehung von Alkali - der Gleichgewichtszustand gestört. Schon die N-Zahlen der I. Periode sind höher. So kommt das weitere Ansteigen nach dem Aussetzen des Alkali weniger deutlich zum Ausdruck, ebenso wie ihr Absinken in der Nachperiode (hohe Zahlen an den beiden ersten Tagen). Aber anch hier ist im ganzen die Neigung zu höheren Zahlen während der mittleren Periode nieht zu verkennen, was auch in den Durchschnittswerthen einigermaassen zum Ausdrucke kommt (Anstieg um $0,7 \mathrm{~g}$ Tabelle IV).

Ziehen wir für die Aufstellung der Stickstoffbilanz auch den Stickstoff der Fäces heran, so ergiebt sich:

Tabelle V.

N-Werthe in Harn und Fäces.

\begin{tabular}{c|c|c|c} 
& T. & W. & Einnahme \\
\hline I. Periode & 18,32 & 15,6 & ca. $14 \mathrm{~g}$ \\
II. $=$ & 17,0 & 14,1 & $=$ \\
III. = & 16,8 & 14,4 & $=$ \\
Ganzer Versuch & 17,3 & 14,2 & $=$
\end{tabular}

Demnach befand sich W. für den ganzen Versuch im Stickstoffgleichgewicht, wahrend T. $3 \mathrm{~g} \mathrm{~N}$ pro die im Durchschnitte verlor. Sein Körpergewicht sank auch von 57,4 auf $55,6 \mathrm{~kg}$. Im 2. Versuche (Tabelle I) dürfte er sich den $\mathrm{N}$-Zahlen des Harnes und dem gleichbleibenden Körpergewicht nach zu urtheilen, im N-Gleichgewicht befunden haben. Da er in beiden Versueben annähernd gleich viel Zucker ausschied, so dürfte für den gesteigerten Eiweisszerfall im 3. Versuche weniger der Zuckerverlust als gestörte Fettresorption verantwortlich zn machen sein. Wenn wir aber der Thatsache begegnen, dass T. in beiden Versuchen zur Zeit der Entziehung des Alkali (Ir. Periode) im Harne nebst höherem Stickstoffgehalte jedesmal auch grössere Zuckermengen aussehied als während der Verabreichung des Alkali, so werden wir trotz gleich hoher Gesammtstickstoffausfuhr in allen 3 Perioden wohl der Vermuthung Raum geben dürfen, dass die dauernde Anwendung von Natriumbicarbonat bei unserem Patienten mit dazu beitrug, einen stärlkeren Eiweisszerfall hintanzuhalten.

Wenn auch, wie bekannt, die Phosphorsäureausscheidung im Harne nicht nur durch die Nahrung, sondern auch noch durch andere 
Bedingungen beeinflusst wird, so ist doch die Beziehung zwischen Kalk- und Phosphorsäureausscheidung bisher allein genauer untersucht worden. Die Ausscheidung beider steht in geradem Verhältnisse, so dass bei verminderter Ca-Aussoheidung durch den Harn auch die $\mathrm{P}_{2} \mathrm{O}_{5}$-Zahl desselben absinkt. . V. Noorden erklärt dieses Verhalten durch die Annahme, dass im Darme befindlicher oder in ihn abgeschiedener Kalk gleichzeitig Phosphorsäure mitreisst. In letzter Zeit wurde ans therapeutischen Gründen versucht, durch übermässige Kalkeinfuhr den Phosphorsäurewerth des Harnes herabzudrücken. Unter solchen Bedingungen muss das Verhältniss von $\mathrm{N}: \mathrm{P}_{2} \mathrm{O}_{5}$ im Harne weiter, bei erhöhter Kalkausfuhr enger werden, in der Norm bei Fleischfütterung beträgt es etwa 8,1:1 (im Muskel $7,6: 1)$.

Auf die Abhängigkeit der Kalkausfuhr im Harne von Alkalescenzverhältnissen wurde von B ertram und Beckman n(l.c.) hingewiesen; beide Autoren sehen die Kalkzahlen des Harnes unter Alkalizufuhr etwas kleiner werden: damit wurde anch das Verhältniss von $\mathrm{N}: \mathrm{P}_{2} \mathrm{O}_{5}$ ein weiteres. In diesem Verhältnisse kommt auch bei unseren Versuchen die Abhängigkeit der Phosphorsäureausfuhr vom Kalkgehalte des Harnes zum Ausdrucke. Die folgende Tabelle (VI) giebt Gelegenheit, die für die ganzen Versuche und für die einzelnen Perioden jedes derselben gewonnenen Durchschnittszahlen und das aus ihnen resultirende Verhältniss von $\mathrm{N}: \mathrm{P}_{2} \mathrm{O}_{5}$ miteinander zu vergleichen.

Tabelle VI.

\begin{tabular}{|c|c|c|c|c|c|c|c|c|c|c|c|c|}
\hline \multicolumn{4}{|c|}{$\begin{array}{l}\text { Durohschnittswerthe fur } \\
\text { den ganzen Versuch }\end{array}$} & \multicolumn{9}{|c|}{ Durchschnittswerthe fur die einzelnen Perioden } \\
\hline & W & 2. Vers. & $\begin{array}{l}\text { T. } \\
\text { 3. Vers. }\end{array}$ & & $\begin{array}{c}\text { W. } \\
\text { II. Per. }\end{array}$ & III. P. & $\begin{array}{r}\text { T. } \\
\text { I. Per. }\end{array}$ & $\begin{array}{l}\text { 2. Versu } \\
\text { III. Per. }\end{array}$ & $\begin{array}{l}\text { uch } \\
\text { III. P. }\end{array}$ & $\begin{array}{c}\text { T. } \\
\text { I. Per. }\end{array}$ & $\begin{array}{l}\text { 3. Versu } \\
\text { II.Per. }\end{array}$ & III. P. \\
\hline $\mathrm{N}$ & 14,151 & 13,973 & 15,702 & 15,543 & 14,001 & 14,085 & 11,587 & 15,234 & 13,837 & 15,399 & 16,041 & 15,325 \\
\hline $\mathrm{Ca}$ & 0,228 & $\begin{array}{l}\text { hoher } \\
\text { Werth }\end{array}$ & 0,709 & 0,155 & 0,276 & 0,217 & Wiedrg. & $\begin{array}{l}\text { hoher } \\
\text { Werth }\end{array}$ & $\begin{array}{l}\text { niedrg. } \\
\text { Werth }\end{array}$ & 0,551 & 0,805 & 0,673 \\
\hline${ }_{2} \mathrm{O}_{5}$ & 1,782 & 2,407 & 2,583 & 1,647 & 1,984 & 1,654 & 2,175 & 2,702 & 1,993 & 2,239 & 2,886 & 2,319 \\
\hline$\frac{\mathrm{N}}{{ }_{2} \mathrm{O}_{5}}$ & 7,941 & 5,805 & 6,079 & 9,437 & 7,057 & 8,515 & 5,32 & 5,64 & 6,94 & 6,88 & 5,55 & 6,6 \\
\hline
\end{tabular}

Danach entspricht das angezogene Verhältniss bei W., sofern es sich um den ganzen Versuch handelt, der Norm (nahe 8:1).

Während Periode I und III liegt es höher, als der Norm entspricht (bei 9,4), während der Entziehung des Alkali um ebensoviel etwa unter der Norm; im ersten Falle wurde also weniger 
Kalk als der Norm entspricht, mit dem Harne ausgeschieden, im 2. Falle mehr als normal. Hier, das heisst in der Periode der Entziehung des Alkali, kommt die bestehende Sänerung ( $3 \mathrm{~g} \mathrm{NH}$ !) auch in den Kalkzahlen einigermaassen zum Ausdruck, deshalb das zu enge Verhältniss von $\mathrm{N}: \mathrm{P}_{2} \mathrm{O}_{5}=7: 1$ ), dort wurde der Einfluss der Säuerung durch die Darreichung von Natr. bic. insoweit wettgemacht, dass ebenso wie beim Normalen unter Alkalizufuhr der Kalkwerth des Harnes noch unter die Norm herabgedrïckt werden konnte (weiteres Verhältniss 9,4:1).

Bei T. ist dieses Verhältniss in beiden Versuchen auf den Durchschnitt berechnet viel enger (6:1), in vollständiger Uebereinstimmung mit seinen absolut hohen Kalkzahlen des Harnes. Gut vergleichbar sind nach dieser Richtung insbesondere der Versuch an W. und Versuch 2 an T., da in beiden Versuchen im Durchschnitte annähernd die gleiche Menge Stickstoff $(14 \mathrm{~g})$ ansgeschieden wurde. Hier finden wir bei W. $1,78 \mathrm{~g} \mathrm{P}_{2} \mathrm{O}_{5}$ pro die, bei T. dagegen $2,4 \mathrm{~g}$, daraus ergiebt sich das angezogene Verhältniss bei ersterem wie $8: 1$ (normal) bei letzterem wie $6: 1$.

In beiden Versuchen an $T$. bleibt das Verhältniss $\mathrm{N}: \mathrm{P}_{2} \mathrm{O}_{\mathrm{i}}$ das gleiche, obwohl in Versuch 214 , in Versuch $315,7 \mathrm{~g} \mathrm{~N}$ pro die ausgeschieden wurden: die Kalkwerthe des Harnes waren offenbar in beiden Versuchen annähernd gleich. In Versuch 3 kommt das Ansteigen der Kalkzahlen bei Entziehung des Alkali auch in diesem Verhältnisse gut zum Ausdrucke (Periode I 6,9, Periode II 5,5, Periode III 6,6); dagegen fehlt diese Uebereinstimmung für die einzelnen Perioden in Versuch 2. Wohl ist das Verhältniss für die II. Periode enger als für die III; dagegen ist es auch für die I. Periode mit ihren verhältnissmässig niedrigeren Kalkzahlen sehr enge eine Abweichung, für die wir eine Erklärung zu geben ausser Stande sind, die aber wohl beweist, das ausser den Ausscheidungsverhältnissen des Kalkes noch andere Bedingungen dieses Verhältniss zu beeinflussen im stande sind, dass also jedenfalls Vorsicht bei Verwerthung dieses Verhältnisses für die Beurtheilung der Menge des mit dem Harne ausgeschiedenen Kalkes geboten ist.

Die Reaction des Harnes blieb bei T. stets saner, trotz Zufuhr der grossen Alkalimenge, eine Thatsache, auf die als Ausdruck mächtiger Säuerung Minkowski hingewiesen hat. Bei W. ging die während der I: Periode alkalische Reaktion nach dem Fortlassen des Alkali sehr rasch in neutrale und stark saure Reaction über, um bei neuerlicher Verabreichung (III. Periode) schon am 2. Tage wieder alkalisch zu werden. Als ziffernmässiger Ausdruck dafür ist 
bei ihm während der I. und III. Periode die als Mononatriumphosphat ausgeschiedene Menge der $\mathrm{P}_{2} \mathrm{O}_{5}$ sehr gering, um in der II. Periode Werthe anzunehmen, die so ziemlich der Norm entsprechen. Bei T. sind diese Werthe stets höher und durch Alkalidarreichnng wenig zu beeinflussen.

Auf die Menge des gebildeten oder richtiger des ausgeschiedenen Aectons und der Oxybuttersäure blieb das Alkali anscheinend ohne EinAluss. Die täglichen Acetonmengen sind sow ohl bei T. als bei W. ungemein wechselnd. Die Menge der täglich ausgeschiedenen Oxybuttersäure kann aus der am Saccharimeter (bei T. nach dem Vergähren) abgelesenen Linksdrehung einigermaassen beurtheilt werden ${ }^{1}$ ). Danach erhielt sie sich bei T. freilich unter vielfachen Schwankungen im ganzen auf gleicher Höhe. Bei W. erreicht sie erst am 13. Tage nach dem Einsetzen der Fleisch-Fettdiät ihren höchsten Werth, um dann dauernd hohe Werthe beizubehalten. Ein Einfluss der Alkalizufuhr auf ihre Menge ist nicht nachweisbar. Diese dürfte bei T., der doppelt soviel Harn, wie die Controlperson ausschied, im ganzen doppelt so hoch gewesen sein wie bei W., an den Tagen grösster Ausseheidung bei jenem etwa 50 , bei diesem $25 \mathrm{~g}$.

Bekanntlich erseheint nur ein geringer Antheil des mit der Nahrung aufgenommenen Kalkes im Harne wieder, der weitaus grössere Theil verlässt den Körper mit dem Fäces, die demnach mit zu untersuchen sind, wo es sich darum handelt, über die Gesammtausscheidung dieser Base ein Urtheil zu gewinnen.

$\mathrm{Zu}$ Beginn einer jeden Periode nnseres 3 . Versuches erhielt $\mathrm{T}$. vegetabilische Kohle in Fr. Müller's ${ }^{2}$ ) Emulsion mit seinem ausgiebigen ersten Frühstücke ( $150 \mathrm{~g}$ Speck und 2 Eier als Omelette).

Bloss zu Beginn der I. Periode wurde die Kohle leider bei nüchternem Magen in Pulverform verabreicht. Die Folge war, wie die Beschaffenheit der nächsten Stïhle lehrte, ein Verstäuben der Kohle im ganzen Darme, was die Abgrenzung der 1. Portion erschwerte. Der Umstand, dass die aus dieser Periode erhaltene Menge der trockenen Fäces unverhältnissmässig gross ist $(188,15 \mathrm{~g}$ gegen 118 in der letzten Periode) und die gewonnenen procentischen

1) Der qualitative Nachweis der Säure in W.'s Harn wurde nach der Külzschen Methode geführt. Der so (aus $1 \frac{1 / 2}{2}$ Litern) Harn gewonnene Aetherextract zeigte starke Linksdrehung $\left(7^{\circ}\right.$ auf $50 \mathrm{ccm}$ Flüssigkeit) und lieferte beim Destilliren mit Schwefelsäure die charakteristischen Krystalle der Crotonsäure.

2) Fr. Müll er, Zeitschr. f. klin. Med. Bd. XlI. 
Ueber die Kalk- und Magnesiaausscheidung beim Diabetes melitus u. s. w. 99

Werthe geben uns allen Grund anzunehmen, dass wir infolge des erwähnten Fehlers für diese Periode noch Kothmengen mit berücksichtigten, die ausserhalb des Versuches fallen. Sonst gelang die Abgrenzung sehr gut, begünstigt durch den Umstand, dass T. täglich regelmässig einen Stuhl absetzte. Bei W., der bloss alle 5-6 Tage einmal zu Stuhle ging, begnügten wir uns damit, die dem ganzen Versuche zugehörigen Fäces abzugrenzen.

Etwa $4 \mathrm{~g}$ des auf dem Wasserbade zar Gewichtsconstanz getrockneten und nach dem Verreiben genau gemischten und gewogenen Kothpulvers wurden mit Salpeter und Natr. carb. im Platintiegel verascht, die Schmelze mit wenig HCl gelöst; von einem ganz geringen Reste, der dabei nicht in Lösung ging - Silicate - wurde abfiltrirt. In der wasserhellen Lösung wurden Kalk and Magnesia, wie im Harne bestimmt letztere hier auch durch Glïhen und Wägen als pyrophosphorsaure Magnesia; da sich das Titriren der aus Filtrat und Waschwasser vom oxalsauren Kalke durch Fällen mit $\mathrm{NH}_{3}$ und Lösen des Niederschlages in Essigsäure gewonnenen Flüssigkeit mit Uranacetat unmöglich erwies.

In je zwei anderen kleineren Portionen des Kothpulvers wurde der Stickstoff nach KjeldahI bestimmt.

In folgender Tabelle (VII) sind die Gesammtausfuhrzahlen für Kalk in Harn und Fäces bei $W$. und für die 3 Perioden bei $T$. zusammengestellt, daneben die daraus sich ergebenden Durchschnittszahlen, sowie die nach König berechnete Kalkzufuhr mit der Nahrung 1). Für T., der etwa 2 Liter Wasser täglich trank, würde sich die tägliche Kalkzufuhr um höehstens $0,1 \mathrm{~g}$ erhöhen.

Tabelle VII.

\begin{tabular}{|c|c|c|c|c|c|c|c|c|c|c|c|}
\hline & & & & & Kalk & & & & & & Kalk \\
\hline & & $\begin{array}{r}\text { Gesamm } \\
\mathrm{T}\end{array}$ & taus & & WY & . & urehseb & aitt $f$ & en $\mathrm{Ta}$ & & \\
\hline & I. Per. & III.Per. & III. P. & Ganz.V. & & 1. Per. & I. Per. & III.P. & anz.V. & & \\
\hline Harn & 2,204 & 6,444 & 693 & 11,331 & 3,419 & 0,551 & 0,805 & 0,673 & 0,666 & 214 & - \\
\hline Fäce & 3,519 & 2,270 & 2,050 & 7,839 & 6,93 & 0,879 & 0,252 & 0,512 & 0,461 & 0,406 & 13,77 i.Ganz. \\
\hline & 5,723 & 8,714 & 4,743 & 19,170 & 10,349 & 1,430 & 1,057 & 1,155 & 1,127 & 0,620 & 0,81 pro die \\
\hline
\end{tabular}

1) $280 \mathrm{~g}$ Fleisch (roh) enthălt 1,2 Proc. Asche, davon 3 Proc. Ca . . 0,1 g 2 Eier $150 \mathrm{~g} \mathrm{Speck}$

$75 \mathrm{~g}$ Butter

$200 \mathrm{~g}$ Spinat

500 Elsässer Wein

$1000 \mathrm{ccm}$ Wasser

$=1,2=\quad$
$=-1=$
$=2=$
$=0,2=$
$=-1=$

$$
\begin{aligned}
& =8==\ldots 0,1= \\
& =-==\ldots 0,05= \\
& =--=\ldots- \\
& =10=\quad \Rightarrow 0,4= \\
& =8==\ldots 0,1= \\
& =0,006=\quad=\ldots 0,06= \\
& 0,81 \mathrm{~g}
\end{aligned}
$$


Aus dieser Zusammenstellung erhellt, dass T. jeden Tag ein halbes g Kalk mehr ausschied, als W., während des ganzen Versuches fast $9 \mathrm{~g}$ mehr als die Controlperson. Da die ausgeführte Menge bei dieser der durch Rechnung gewonnen eingeführten halbwegs entspricht, müssen wir wohl annehmen, dass T. eine fast eben so grosse Kalkmenge verlor (unter Berücksichtigung mehr eingeführten Wassers zu mindest 0,4 pro die, 6,8 im ganzen $\left.{ }^{1}\right)$ ).

Fragen wir nach dem Herkommen dieser enormen Kalkmengen, so werden wir wohl ebenso wie $\nabla$. Noorden ${ }^{2}$ ) auf Zerfall von Knochen hingewiesen, da T.'s täglicher Verlust an Muskelfleisch während dieses Versuches $\mathrm{zu} 75 \mathrm{~g}(3 \mathrm{~g} \mathrm{~N})$ berechnet, bloss einer. Mehrausgabe von $0,02 \mathrm{~g}$ CaO per Tag entsprechen wiirde. Wenn wir nun auch v. Noorden's Bedenken, aus dem Engerwerden des Verhältnisses von $\mathrm{N}_{2} \mathrm{P}_{2} \mathrm{O}_{5}$ im Harne allein schon auf Knochenzerfall zu schliessen, theilen, so ist doch nochmals hervorzuheben, dass in unserem Falle Engerwerden dieses Verhältnisses mit Knochenzerfall einherging. Wir wollen nicht unterlassen, an dieser Stelle der Bemerkung v. Frerieh's ${ }^{3}$ ) zu gedenken, dass Schultzen die Knochen von Diabetikern untersucht und sie abnorm leicht gefunden habe.

Es liegt nahe, die Ursache der Auflösung des Knochenkalkes auf die lange Finwirkung organischer Säuren zu beziehen und auf den Alkalimangel der Gewebe, der dadurch zustandekommt.

Die negativen Ergebnisse aber, die $\mathrm{H}$ eiss ${ }^{4}$ ) bei Fütterung mit Milchsäure hinsichtlich der Vermehrung des Kalkes erhielt, erklären sich, wie schon gesağt, ohne Mühe dadurch, dass diese organische Säure noch verbrannt wurde, ehe sie mit Knochen in Berührung kam, und also nicht geeignet erscheint, Alkalimangel zu erzengen. Anderseits ist die Thatsache, dass W., obwohl er gleichfalls namhafte Mengen von Oxybuttersäure ausschied, gleichwohl nicht wesentlich kalkärmer wurde, in dem Sinne zul denten, dass der Organismus die Fähigkeit besitzt, seinen Alkalibestand eine Zeitlang zu wahren - vorerst unter Heranziehung des Ammoniaks und dass erst lange währende Säuerung seinen Bestand an dem schwerer verfügbaren Knochenkalke angreift.

1) Auch Moraczewski findet bei seinem schweren Diabetiker ein Kalkdeficit.

2) v. Noorden, loc. cit. S. 416.

3) v. Frerich, Diabetes. Berlin 1884 ,

4) Heiss, Zeitschr. f. Biol. Bd. XII. S. 151. 
Mit Rücksicht auf die Grösse der Kalkausfuhr bei W. făllt ohne weiteres der Einwand weg, das die vermehrte Kalkausfuhr bei T. auf zu geringen Kalkgehalt der Nahrung zu beziehen sei; For ster ${ }^{1}$ ) fand nach Verfütterung von kalkarmem Futter bei Hunden, dass Kalk von den Knochen abgegeben wurde. Darnach fanden Fr. Mü 11 er $^{2}$ ) und $P$ erl ${ }^{3}$ ), dass auch bei reiner Fleisehfettnahrung mehr Kalk in Harn und Fäces aus geschieden wurden, als der Einfuhr entsprach. In nnserem Falle genügte, wie das Verbalten der Controlperson zeigte, der dazu noch mit dem Gemüse, Wein und Wasser zugeführte Kalk, um beim normalen Individuum den Kalkbestand des Organismus zu erhalten.

Das weitaus grösste Interesse scheint uns ein Vergleich der Kalkwerthe in Harn und Fäces zu bieten.

Wie erwähnt, ist der Darm in der Norm die Hauptausscheidungsstätte fur den Kalk. v. Noorden und Belgardt) fanden das Verhältniss der beiden etwas wechselnd, im Durehschnitte wurden bloss 10 Proc. des Gesammtkalkes mit den Nieren, 90 Proe. mit den Fäces ausgeschieden; der kleinste Werth für letzteren betrug 71 Proc. Aehnlich lauten die Angaben anderer Autoren 5) für Menseh und Hund (Fleischfresser). Der Pflanzenfresser scheidet gar nur wenige Procente seines Kalkes mit dem Harne aus. Gleiches Verhalten ergaben die Versuche von Rey ${ }^{6}$ ) und Tereg und Arnold ${ }^{7}$ bei subeutaner Injection von essigsaurem Kalke. Ersterer fand bloss 26 Proc. des eingeführten Kalkes im Harne, bis 53 Proc. im Kothe wieder. Auch bei letzteren war die Kalksteigerung im Harne gering. Bei Rüdel betrug sie gar nur 12 Proc. des eingeführten Salzes, im Vergleiche mit unserem Versuche aber ist bemerkenswerth, dass Forster (1. c.) auch bei dem Knochenzerfalle infolge von Zufubr kalkarmen Futters die Hauptmasse des Skelettkalkes in den Fäces fand. Ebenso schied der Hungerhund Etzinger's s) 0,074 CaO mit dem Harne, 0,14 mit den Fäces aus.

1) Forster, Zeitschr. f. Biol. Bd. XII. S. 464.

2) Fr. Müller, Zeitschr. f. Biol. Bd. XX. \$. 358 .

3) Per1, Virch. Archiv Bd. LXXIV. S. 54.

4) v. Noorden und Belgardt, Berl. klin. Wochenschr. 1894. Nr. 10.

5) Forster 17,8:82,2 (Mensch), Fleitmann 28,7:71,3 (Mensch), Bertram $43,3: 60,4$ (Mensch). (S. Bertram l. c. Tabelle.)

6) Rey, Archiv f. experiment. Pathol. u. Pharmak. Bd. XXXV. S. 297.

7) Tereg und Arnold, loc. cit.

8) Etzinger, Zeitschr. f. Biol. Bd. X (cit. nach Voit, Physiol. des Stoffw.). 
Aus Tabelle VII. erhellt ohne weiteres, dass dieses Verhältniss, zunächst für T. eine völlige Umkehrung erlitt. Für den ganzen Versuch schied er im Harne 59 Proc., in den Fäces 41 Proc. des Gesammtkalkes aus. Während der 2. Periode mit ihren hohen Kalkzahlen, wo die Säuerung unbeeinflusst durch Alkalidarreichung voll zur Geltung kam, ist die Zahl für den Kalk der Fäces ganz besonders niedrig; sie entspricht bloss 23 Proc. der Gesammtausscheidung. Bloss in der ersten Periode entspricht sie anscheinend einigermaassen den gewöhnlich vorgefundenen Verhältnissen; nach dem, was wir aber über die mangelhafte Kothabgrenzung gerade während dieser Periode gesagt hahen, werden wir gut thun, den Werth für die Fäces um $1 / 3$ zu erniedrigen, darauf hinweisend, dass ftir die I. und III. Periode der Kalk etwa gleich viel Proc. der Trockensubstanz ausmachte (1,87 und 1,74 Proc. gegen 1,2 Proc. für die 2. Periode). Nehmen wir diese Correctur vor, so ergiebt sich, dass T. während aller 3 Perioden gleichviel Kalk ausschied, wenn wir Harn und Fäces zusammennehmen, eher wurde während der Alkalientziehung ein etwas kleinerer Werth im ganzen festgestellt. Dureh die Alkalidarreichung wurden demnach die Kalkzahlen bloss zu Gunsten der Fäces verschoben. Ein Vergleich mit Bertram's Versuch mit $40 \mathrm{~g}$ citronensaurem Kali zeigt vollständige Uebereinstimmung. Der Kalkgehalt der Fäces stieg bei ihm von 0,698 auf 0,875 , während sich der entsprechende Werth im Harne von 0,500 auf 0,285 ermässigte. Die Summe betrug im 1. Falle 1,198, im 2. $1,16 \mathrm{~g}$.

Wenn wir nun auch unterlassen baben, bei $W$. die Fäces nach einzelnen Perioden abzugrenzen, so werden wir doch nicht mit der Annahme fehl gehen, dass auch bei ihm hohem Kalkwerthe des Harnes niedriger Kalkwerth der Fäces entsprochen habe; demnach dürfte an dem Tage, da er $0,4 \mathrm{~g}$ Ca mit dem Harne ausschied, bei einer durchschnittlichen Gesammttagesmenge von $0,6 \mathrm{~g}$ wohl auch bei ihm eine Umkehrung des gewöhnlichen Verhältnisses stattgehabt haben.

Um ein Verständniss dieses sonderbaren Befundes anzubahnen, wollen wir den Weg verfolgen, den der mit der Nahrung eingeführte Kalk zurückzulegen hat. Während man früher annahm, dass bloss der mit dem Harne ausgeschiedene Kalk der Resorption anheimgefallen ist, weiss man vor allem durch die Arbeit Forster's ${ }^{1}$ ), dass der Darm nicht nur der Resorption, sondern auch der Secretion

1) Forster, Archiv f. Hygiene Bd. II. S. 385. 
des Kalkes dient. Auf den Versuchen Wildt's fussend, der bei Pflanzenfressern $3 / 4$ des Nahrungskalkes im Darme erst resorbirt und erst nachträglich zu $2 / 3$ wieder in den Darm ausgeschieden fand, findet Forster auch beim Hunde, der 4 Stunden nach der Mahlzeit getödtet worden, bis 59 Proo. des eingeführten Kalkes resorbirt. Als Ausdruck wieder beginnender Secretion in den Darm zeigte der Kalkgehalt des Darminhaltes vom Magen bis zum Ileum in den einzelnen abgebundenen Partien wachsende Werthe. Den endgültigen Beweis für nachträgliche Kalksecretion in den Dickdarm erbrachte in neuester Zeit Rey ${ }^{1}$, der auch nach Unterbindung des Dünndarmes 22 Proe. des eingeführten Kalkes im Dickdarme wiederfand. Beim hungernden Hunde beträgt der Kalk des Dickdarminhaltes gar 86 Proc. der ganzen im Darmkanale auffindbaren Menge.

Aus diesen Thatsachen erhellt ohne weiteres, dass das Ver. hältniss des Kalkes in Harn und Fäces sowohl von der Grösse der Resorption als von der Grösse der nachträglichen Secretion in den Darm abhängig ist. Ist erstere geschädigt, wie im Typhus (S chetelig l. c.), oder bei Dünndarmkatarrh (Forster), muss dies die Menge des Harnkalkes herabdrücken. Den gleichen Erfolg hat die Verabreichung von citronsaurem Kali (Bertram) oder von Natriumbicarbonat, unter deren Einfluss die Ausscheidung in den Darm offenbar gesteigert ist. Gegenwart organischer Säuren im Blate, die mit dem Kalke leicht lösliche Verbindungen eingehen, und gleichzeitiger Alkalimangel werden umgekehrt die Kalkausscheidung in den Diekdarm beeinträehtigen, was eine Aenderung des Verhältnisses von Harnkalk zum Kalke der Fäces zu Gunsten des ersteren zur Folge hat, wie dies in unserem Versuche deutlich zum Ausdrucke kommt.

Wenn wir uns aber daran erinnern, dass auch in den Versuchen an den Hungerkünstlern Cetti und Breithaupt ${ }^{2}$ ) eine Aenderung: des gewöhnlichen Verhältnisses, genau wie in unserem Versuche, sich ergab - Cetti schied 3,91 g CaO mit dem Harne, 0,97 g mit den Fäces aus - so dürfen wir wohl der Vermuthung Raum geben, dass auch hierfür der Alkalimangel einerseits und das Auftreten abnormer Säuren andrerseits verantwortlich zu machen war. Cetti schied reichlich Aceton und Acetessigsäure aus.

1) Rey, Archiv f. experiment. Pathol, u. Pharmakol. Bd. XXXV. S. 295.1895.

2) L.eh mann, Müller u. s. w., Virch. Arch. Suppl, zu Bd. CXXXI, 1893. 
Im 3. Versuche (T. und W.) wurde auch die Magnesia in den Kreis der Beobachtung gezogen. Ein Blick auf Tabelle II und III beweist, dass ihre Ausscheidung im Harne mit der des Kalkes durchaus nicht parallel lief. In allen Perioden finden wir bei beiden Kranken annähernd gleich hohe Zahlen (um 0,25 g pro die 1). Tab.VIII giebt die absoluten mit Harn und Fäces ausgeschiedenen $\mathrm{MgO}$-Mengen und die Durchschnittswerthe, auf den Tag berechnet.

Tabelle VIII. MgO-Werthe.

\begin{tabular}{|c|c|c|c|c|c|c|c|c|c|c|c|}
\hline & & & & & 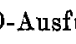 & & & & & & $\mathrm{MgO}-\operatorname{Ein}-$ \\
\hline & & Gesamn & $\begin{array}{l}\text { tausfuh } \\
\text {. }\end{array}$ & & & & Im Dur & $\begin{array}{l}\text { chschnit } \\
\text { c. }\end{array}$ & pro die & & \\
\hline & I.Per. & Per. & III. P. & Ganz.V. & & I.Per. & II.Per. & III. $\mathrm{P}$. & Ganz.V & & \\
\hline Harn & 0,985 & 2,320 & 1,257 & 4,562 & 3,611 & 0,244 & 0,258 & 0,314 & 0,272 & 0,202 & - \\
\hline Fäces & 1,374 & 1,260 & 1,159 & 3,793 & 1,699 & 0,343 & 0,140 & 0,290 & 0,233 & 0,1 & - \\
\hline Zus. & 2,359 & 3,580 & 2,416 & 8,355 & 5,310 & 0,589 & 0,398 & 0,604 & 0,505 & 0,312 & 0,31 \\
\hline
\end{tabular}

Die Werthe für die Fäces der I. Periode bei T. wurden in der Tabelle um ein Drittheil erniedrigt. Wir verweisen wieder auf das oben über die Abgrenzung der Fäces dieser Periode Gesagte und auf die procentualischen Werthe auf Tabelle II, durch die uns solehe Correctur gerechtfertigt erscheint. Darnach schied W. im Durchschnitte so viel Magnesia aus, als er mit der Nahrung einnahm, entsprechend seinem Verhalten zum Kalke, T. darüber hinaus, 3,045 oder $0,193 \mathrm{~g}$ für jeden Tag. Von den $3 \mathrm{mg}$ absehend, die auf Rechnung seines Eiweissverlustes (75 g Muskelfleisch pro die) zu setzen sind, ergiebt sich eine tägliche Mehrausgabe von $0,193 \mathrm{~g}$ $\mathrm{Mg0}$, also fast ein Dritttheil des Gewichtes des täglichen Kalkverlustes $(0,507)$, was mit der Annahme von Knochenzerfall vollauf übereinstimmt.

Das Verhältniss der Ausscheidung der Magnesia in Harn und Koth ist in der Norm dem beim Kalke beobachteten entgegengesetzt.

1) Gaethgens und Beckmann (loc. cit.) vermissten gleichfalls eine Beeinflussung der Mg-Ausfuhr mit dem Harne durch Săuren oder Alkalien.

2) Berechnet nach König:

$280 \mathrm{~g}$ Fleisch enth. 1,2 Proc. Asche, davon 3,21 Proc. MgO . . 0,11 g

\begin{tabular}{|c|c|c|c|c|c|c|c|c|c|}
\hline 2 Eier & $=$ & 1,2 & $=$ & $=$ & $=$ & 2,43 & $=$ & $=$ & $.0,03=$ \\
\hline $50 \mathrm{~g} \mathrm{Speck}$ & $=$ & - & $=$ & $=$ & $=$ & - & $=$ & $=$ & . . $0,05=$ \\
\hline $200 \mathrm{~g}$ Spinat & $=$ & 2,0 & $=$ & $=$ & $=$ & 2,3 & $\approx$ & $=$ & . $0,09=$ \\
\hline 600. ccm Wein & $=$ & 0,2 & $=$ & $=$ & : & 2,7 & $=$ & $\equiv$ & $\therefore \frac{0,03=}{0,31 \mathrm{~g}}$ \\
\hline
\end{tabular}


Ueber die Kals- und Magnesiaausscheidung beim Diabetes melitus u. s. w. 105

60 Proc. erscheinen im Harne, 40 Proc. im Kothe. Bei W. bleibt dieses Verhältniss erhalten, bei T. werden im Harne und Kothe etwa gleich grosse Mengen der Base ausgeschieden.

Während in der Norm die Kalkmenge des Harnes halb so gross ist wie sein Gehalt an Magnesia, scheidet T. mit dem Harne in der I. und III. Periode etwa das Doppelte, während der II. Periode dreimal soviel Kalk als $\mathrm{Mg}$ aus. Ein ähnliches Verhalten finden wir bei W. an den Tagen hoher Kalkausscheidung (27. Febr. auf Tab. III.), - ein weiterer Beweis dafür, dass die Kalkzahl an diesem Tage höher war, als bei einer sonst im Gleichgewichte befindlichen Person zu erwarten gewesen wäre ${ }^{1}$ ). Am auffallendsten bleibt der procentualiseh und absolut niedrige Werth für die Magnesia in den Fäces während der alkalifreien Periode bei T. - , die eine Erniedrigung der Gesammt-Mg-Ausfuhr dieser Periode, um täglich $0,2 \mathrm{~g}$ zur Folge hat. Wir werden wohl die Annahme nicht umgehen können, dass unter dem Einflusse des Alkalimangels ein Theil der aus zerfallenen Knochen frei gewordenen Magnesia im Organismus zuruekbehalten wurde.

Wir erinnern daran, dass auch in Gaethgens (1. e.) Versuche mit Schwefelsäurezufuhr einer vermehrten Kalkausscheidung ein Herabgehen des Magnesiawerthes entsprach.

Es erübrigt noch, einige Worte über das Auftreten von $\beta$-Oxybuttersäure bei dem Nichtdiabetischen (W.) zu sagen. Ausser bei Diabetes wurde sie von $\mathrm{Külz}^{2}$ ) und anderen im Harne bei fieberhaften Infeetionskrankheiten aufgefunden. In einem Falle von Scorbut stellte Minkow ski3) $3 \mathrm{~g}$ der Sänre aus dem Harne dar. In geringer Menge begleitet sie gelegentlich chronische Inanitionszustände ${ }^{4}$, zumal bei Krebs (K lemperer). In allen diesen Fällen war ibr Auftreten an reichlichen Zerfall von Organeiweiss geknüpft, wie v. Noorden ${ }^{5}$ ) ausdrücklich hervorhebt.

Weintrau $d^{6}$ ) wies zuerst Oxybuttersäure bei einem Diabetiker

1) Diese Umkehrung des Verhältnisses von $\mathrm{Ca}$ und $\mathrm{Mg}$ zeigte auch der schwere Diabetiker Neubau er's (l. c.).

2) Kitla, Zeitschr. f. Biol, Bd. XXIII. S. 336 .

3) Minkowski, Archiv f. experiment. Pathol. u. Pharmakol. Bd. XIX. S. 225.

4) In einem Falle von hysterischem Erbrechen konnte der Eine von uns vor kurzem gegen $40 \mathrm{~g}$ in der Tagesmenge nachweisen.

5) v. Noorden, l. c. S. 220.

6) Weintraud, Archiv f, experiment. Pathol. u. Pharmakol. Bd. XXXIV. S. 169 . 
nach, der sich im Stickstoffgleichgewichte befand. Es war das unser Patient T. Letzteres bestand, wie wir wahrseheinlich zu machen suchten, während unseres 2. Versuches.

Bei W. war es wohl.während des ganzen Versuches nicht gestört. Gleichwohl war die ausgeschiedene Menge der Säure, wie die Zahlen für die Linksdrehung des Harnes und des Aetherextractes zeigen, sehr beträchtlich — zumindest im Vergleiche mit jenen Mengen, die bislang bei Nichtdiabetischen aufgefunden werden konnten. Es geht demnach nicht an, bei unserem Kranken W. Zerfall von Körpereiweiss für ihr Auftreten verantwortlich zu machen.

Näher lag es, den Ausschluss aller Kohlehydrate bei der Nahrung zur Erklärung heranzuziehen, nachdem durch die Arbeiten von Rosenfeld 1) und Hirschfeld ${ }^{2}$ ) festgestellt wurde, dass reine Fleisch- oder Fleischfettdiät - wie im Hunger - Aceton und Acetessigsäure im Harne in vermehrter Menge erscheinen lässt, also Körper, deren nahe chemische und biologisehe Beziehung zur Oxybuttersäure genügend feststeht, und deren starke Vermehrung in unserem Falle uns zuerst veranlasst hatte, nach Oxybuttersäure zu suchen.

In einem je achttägigen Versuche nahm jeder von uns die gleiche Nahrung ein wie unsere Patienten. Bei uns beiden war sohon am 3. Tage intensive Eisenchloridreaction neben Aceton im Harne nachweisbar. Beim einen am 5., beim anderen am 7. Tage zeigten unsere Harne im Polarimeter eine Linksdrehung von 0,3 bis $0,4^{\circ}$. Der Alkoholätherextract zeigte gleichfalls intensive Linksdrehung und lieferte beim Destilliren mit Schwefelsäure Crotonsäure. Es besteht demnach wohl kein Zweifel, dass auch in unseren Harnen Oxybuttersäure vorhanden war, wenn auch in geringerer Menge, als bei W., und dass ihr Auftreten durch die eigenthümliche Diät begünstigt wurde.

Hirsehfeld (1. e.) vermisste Aceton im Harne bei Unterernährung mit Fleisch- und Kohlehydraten, desgleichen bei ausschliesslicher Ernährung mit Fleisch ${ }^{3}$ ); er sah es aber auch dann auftreten, wenn dem Körper eine genügende Calorienmenge in der Form von Fleisch und Fett zugeführt wurde. Aehnlich lagen die

1) Rosenfeld, Deutsch. med. Wochenschr. 1885. S. 683 .

2) Hirschfeld, Zeitschr. f. klin. Medicin Bd. XXVIII. S. 176.

3) In einer anderen Arbeit (Deutsch. med. Wochenschr. 1893, S. 914) setzt er die acetonzerstörende Wirkung von $100 \mathrm{~g}$ Eiweiss der Nahrung der von $20-40 \mathrm{~g}$ Kohlehydraten gleich. 
Verhältnisse in unseren Versuchen. Die zugeführte Eiweissmenge stand nahe der unteren Grenze jener Werthe, deren Zuführung von den Autoren als für die Erhaltung des Körpereiweisses nothwendig bezeichnet wird.1) Sie berechnet sich mit $1,25 \mathrm{~g}$ Eiweiss pro Kilogramm Körpergewicht. Die darüber hinaus nöthigen Calorien wurden in der Form von Fett eingebracht.

Demnach glauben wir uns zu dem Schlusse berechtigt, dass bei W. ebenso wie bei uns das Auftreten von Oxybuttersäure, Aceton und Acetessigsäure bei einer Nahrung, die uns eben noch im Stickstoffgleichgewichte erhielt, von zwei Einflüssen abhing. Einmal fehlten in der Nahrung die Kohlehydrate, deren die Oxydation begünstigender Einfluss bekannt ist. Anderseits stand auch ans dem Eiweisse stammendes Kohlehydrat dem Stoffwechsel nicht in genügendem Maasse zur Disposition. Freilich ist die Möglichkeit, dass im Verlaufe der 24 Stunden des Tages vorübergehend Stickstoffdeficit und Einschmelzung von Körpereiweiss stattgefunden hat(v. No orden), nicht zurtuckzuweisen. Die Unterschiede in der Menge der bei uns und bei $W$. ausgeschiedenen Säure müssen wir wohl auf individuelle Verschiedenheiten der Personen zurückftuhren.

Im Folgenden stellen wir kurz die wichtigsten Ergebnisse unserer Arbeit zusammen.

1. Die Ausscheidung des Kalkes im Harne geht beim Gesunden und beim Diabetiker der Ammoniakansscheidung parallel und ist wie diese dureh Alkalizufuhr herabdrückbar.

2. Unter dem Einflusse abnormer Säurebildung (Acidose) nimmt die Kalkausfuhr mit dem Harne besonders hohe Werthe an.

3. Durch Säuerung im Organismus bedingte Verarmung an Alkali führte bei dem zu unserem Versuche gewählten schweren Diabetiker zu Knochenzerfall mit vermehrter Ausscheidung von Kalk und Magnesia.

4. Unter dem Einflusse abnormer Säurebildung ändert sich das gewöhnliche Verhältniss der Ausscheidung des Kalkes im Harne und in den Fäces in dem Sinne, dass die Hauptmasse des Kalkes mit dem Harne ausgeschieden wird.

1) S. von Noorden, Pathologie des Stoffwechsels. 
108 VI. GerHardT u. SchLesinger, Ueber Kalk- u. Magnesiaausscheidung u.s.w.

5. Bei Alkalimangel scheint Magnesia im Organismus zurückgehalten zu werden.

Bei unseren Versuchen ergab sich als Nebenbefund:

6. Bei kohlehydratfreier Nahrung und knapp bemessener Eiweisszufuhr tritt beim Gesunden anscheinend regelmässig neben Aceton und Acetessigsäure Oxybuttersäure auf, und zwar auch bei erhaltenem Stickstoffgleichgewichte.

Strassburg i. E., den 24. December 1898. 